\title{
XXXIII. Some new mechanical quadratures
}

\section{George F. Becker}

To cite this article: George F. Becker (1911) XXXIII. Some new mechanical quadratures, Philosophical Magazine Series 6, 22:128, 342-353, DOI: 10.1080/14786440808637130

To link to this article: http://dx.doi.org/10.1080/14786440808637130

曲 Published online: 20 Apr 2009.

Submit your article to this journal $\pi$

Џll Article views: 2

Q View related articles $\asymp$

4 Citing articles: 1 View citing articles 


\section{Conclusion.}

$\$ 17$. We were already led by our first investigations to the opinion that the further one was able to penetrate into the infra-red, the simpler the relations would become. While we had to limit ourselves in 1893 to the region

$$
2<b^{\prime} / \lambda<75,
$$

the results in the present communication cover the interval

$$
0.08<b^{\prime} / \lambda<2 \text {; }
$$

if we consider the measurements for $\lambda=100 \mu$ carried out with an angle of inclination $\beta=60^{\circ}, b^{\prime} / \lambda$ even becomes equal to zero.

The problem which we set ourselves in the beginning may therefore be considered as solved. Indeed, the general result throughout corresponds to expectation, in that a practically complete Hertzian polarization finally occurred with all gratings $(\$ 10)$.

Our final result, which assumes quite a simple form, is throughout in unison with the principles of the electromagnetic theory of radiation. The special theories mentioned $j_{n}$ the introduction also show the same final result, provided that $b / \lambda$ or $d / \lambda$ be only sufficiently small. The theories are still unsatisfactory with regard to the extended interval between; the relations there are much more complicated even, although they again simplify themselves in the firstmentioned optical region.

We are much indebted to Mr. Morris Owen for help in preparing this paper.

\section{Some New Mechanical Quadratures. By George F. Beck er *.}

$M^{1}$ ECHANICAI quadratures are in some circumstances unavoidable, but they are usually shumned as clumsy and troublesome. Were the formulæ neater and their applicability better defined, they might be of great use in experimental physics and might compete with other integrations by series in the computation of functions. This paper is intended as a contribution to that end.

There are three distinct systems of mechanical quadrature, each depending upon the integration of a general formula for interpolation. By integrating Newton's interpolition formula, Simpson's rule, Weddle's rule and some others can

* Communicated by the Author. 
$b_{e}$ found. These depend upon the assumptions that the increments of the abscissa are constant and that the differences above a certain order are negligible. It is said that if the attempt is made to include in quadratures of this species differences above the sixth, the formulæ become unmanageable, but this I have not tested. It is possible to base quadratures on Stirling's and Bessel's interpolation formulas, but as these are not applicable at the beginning or the end of a ser.es of values their usefulness is limited, and since these modes of interpolition are founded on Newton's, the quadratures are not essentially different.

Gauss used as the basis of his method of quadratures Lagrange's interpolation formula. When after integration the increment of the abscissa is assumed to be constant, Cotes'a numbers result, but Gauss showed that, by a proper selection of unequal increments of the independent variable, formulis can be deduced whose convergence is very rapid. Unfortunately most of the increments are surds, renderings the application of the method very laborious in spite of its elegance.

Interpolation by means of Taylor's series has several advantages over methods depending upon finite differences, and its limitation to continuous functions is rarely of any moment. In dealing with known functions, the methods of infinitesimal calculus are habitually employed excepting for interpolation, while finite differences constitute a distinct algorithm. Since Taylor's series is the very foumdation of analysis, its application to interpolation is both more consistent and inore elegant than that of finite differences, while, if needful, the final results can be expressed in terms of finite differences without the least truuble. Similarly, so-called mechanical quadratures founded on Taylor's series in its application to interpolation are quadratures obtained by integration of a differential equation, and thus do not differ essentially from integrations by series. The expressions for quadrature to which Taylor's theorem leads are in some cases semi-convergent series, yet the error involved may be reduced ad lilitum. Although convergent series would yield results of absolute accuracy were an infinite number of terms to be computed, this accuracy is purely theoretical and computation terminates when the error becomes negligible. The distinction between convergent and semi-convergent series is clear, yet there is no difference between the results obtainable by their use in effecting quadratures.

The quadrature founded on Taylor's series was first given 
by Euler in 1732-3, but his paper was not printed until 1738. It was discovered independently by Maclaurin, who published it in $1742^{*}$. The very essential discussion of the remainder was left to Poisson, Jacobi and others. The formula for mechanical quadratures commonly given in t.extbooks, and ordinarily ascribed to Laplace, is merely Euler's equation with the substitution of finite differences for derivatives.

It is easy so to transform Taylor's series as to express an integral in terms of a sum of the ordinates and sums of the successive derivatives. The function to be integrated and its derivatives can be similarly expressed. From the system of equations thus developed, the sums of the derivatives can be eliminated and the result taken between limits is a definite integral expressed in terms of the sum of the ordinates, together with the derivatives at the limits. This is Euler's quadrature.

Let $h$ be the constant increment of $x$ and $B_{1}, B_{3}, B_{5} \ldots \ldots$ be Bernoulli's numbers ; for brevity let also

$$
v^{k}=f\left(x_{n}^{(k)}\right)-f\left(x_{0}\right)
$$

or the difference of the $k$ th derivative at the two limits.

Then Euler's equation may be written thus :

$$
\begin{aligned}
& \int_{x_{0}}^{x_{n}} y d x=h\left(\frac{y_{0}}{2}+y_{1}+y_{2}+\ldots+\frac{y_{n}}{2}\right)-\frac{\mathrm{B}_{1} h^{2} v^{\prime}}{2 !}+\frac{\mathrm{B}_{3} h^{4} v^{\prime \prime \prime}}{4 !}-\ldots+\mathrm{R} . \\
& \text { Of } \operatorname{course} \quad h=\frac{x_{n}-x_{0}}{n}
\end{aligned}
$$

and $h$ may be any integral factor of $x_{n}-x_{0}$. The total number of ordinates is $n+1$ and they divide the area to be integrated into $n$ parts each of width $h$.

Euler's formula is capable of some transformations which do not seem to have been noticed and a variety of special forms can be deduced from it. For this purpose it is convenient to make certain changes in notation. The first term of the second member is a polygon bounded by the axis, the extreme ordinates and chords connecting the extremities of all the ordinates. Let this polygon of chords be denoted by

* Euler's formula, based on Taylor's theorem, will be found in Commentarii Acad. Sci. Imp. Petrop. vol. vi. ad monos 1732 et 1733; 1738, page 68; Maclaurin gives it in his 'Treatise of Fluxions,' 1742, page $67:$. 
$\mathrm{C}_{1}$ and let

$$
\begin{aligned}
& \mathrm{C}_{2}=2 h\left(\frac{y_{0}}{2}+y_{2}+y_{4}+\ldots \cdot \frac{y_{n}}{2}\right) \\
& \left.\ldots=\ldots \ldots \ldots \ldots . . . \cdots+\frac{y_{n}}{2}\right), \\
& \mathrm{C}_{m}=m h\left(\frac{y_{0}}{2}+y_{m}+y_{2 m}+\ldots .\right.
\end{aligned}
$$

in which case $n$ must be divisible by $m$. Evidently these are also polygons of chords and there is an Eulerian equation corresponding to each of them, obtainable by merely substituting $m h$ for $h$. Let also

$$
\mathrm{T}=2 h\left(y_{1}+y_{3}+y_{5} \ldots+y_{n-1}\right)=2 \mathrm{C}_{1}-\mathrm{C}_{2} \text {. }
$$

Here $\mathrm{T}$ may be a polygon of tangents or of tangents with portions of the ordinates. It consists of $n / 2$ portions each of width $2 /$. In any case the integral songht will be an area intermediate between $\mathrm{T}$ and $\mathrm{C}_{n}$ irrespective of the particular value of $m$. It is evident from the identity $\mathrm{T} \equiv 2 \mathrm{C}_{1}-\mathrm{C}_{2}$ that

$$
\int_{x_{0}}^{x_{n}} y d x=\mathrm{T}+\left(2^{2}-2\right) \frac{\mathrm{B}_{1} h^{2} v^{\prime}}{2 !}-\left(2^{4}-2\right) \frac{\mathrm{B}_{3} h^{4} v^{\prime \prime \prime}}{4 !}+\ldots+\mathrm{R} \text {. }
$$

Suppose $n$, or the number of strips into which the area is divided, to be a multiple of two. 'Then the integral is expressible by each of two or more Fulerian equations. If each of these is multiplied by an arbitrary multiplier and if the sum of these multipliers is unity, the sum of the equations will be a new expression for the integral. Furthermore, for every polygon of chords involved it will be possible to eliminate the coefficient of one difference of derivatives, or $v$. Thus if $n=4$ the integral may bo expressed in terms of $\mathrm{T}, \mathrm{C}_{2}$, or $\mathrm{C}_{4}$. Multiplying by arbitrary coefficients and adding the three equations makes it possible to impose three conditions : viz., that the sum of the multipliers shall be unity and that the coefficients of $v^{\prime}$ and $v^{\prime \prime \prime}$ shall disappear. 'This transformation leaves the integral expressed in terms of the three polygons and of derivatives of the fifth and bigher orders for which finite differences may be substituted if necessary.

So far as mere elimination is concerned there appears to be no limit to this process. Thus if $x_{n}-x_{0}$ were to be divided into 60 parts, eleven coefficients could be eliminated and the integral would be expressed in terms of $\mathrm{T}$ and eleven polygons of chords, only the $23 \mathrm{rd}$ and higher derivatives or 
differences appearing in the remaining portion of the series. For certain classes of functions this might be advantageous, but on account of the semi-convergence of Euler's series the desirable limit will in many instances be lower.

I have carried out the process only as far as $n=12$, which permits of the elimination of all the derivatives below the eleventh. The equations themselves show the appropriate factors, while the coefficient of the one derivative term retained is the sum of the coefficients in the several Euler series each multiplied by one of these factors. The following six formulas are thus obtained :-

(1)

$\int^{*}$

$$
\begin{aligned}
& =\frac{2 \mathrm{~T}+\mathrm{C}_{2}}{3}-2 h \frac{h^{3} v^{\prime \prime \prime}}{360}+\ldots \\
& =\frac{32 \mathrm{~T}+12 \mathrm{C}_{2}+\mathrm{C}_{4}}{4 !}-4 h \frac{h^{5} v^{\top}}{1890}+\ldots \\
& =\frac{648 \mathrm{~T}+81 \mathrm{C}_{2}+112 \mathrm{C}_{3}-\mathrm{C}_{6}}{840}-6 h \frac{h^{7}{ }^{\mathrm{vii}}}{5,600}+\ldots \quad(n>6) \\
& =\frac{2,048 \mathrm{~T}+704 \mathrm{C}_{2}+84 \mathrm{C}_{4}-\mathrm{C}_{8}}{2,835}-8 h \frac{2 h^{7} v^{\mathrm{vii}}}{4,725}+\ldots \quad(n>8) \\
& =-\frac{35,000 \mathrm{~T}+14,375 \mathrm{C}_{2}+528 \mathrm{C}_{5}-7 \mathrm{C}_{10}}{49,896}-10 h \frac{10 h^{7} u^{\mathrm{vii}}}{12,09 b^{\circ}}(n>10) \\
& =\frac{1,492,992 \mathrm{~T}-174,960 \mathrm{C}_{2}+585,728 \mathrm{C}_{3}}{1,801,800} \\
& -\frac{104,247 \mathrm{C}_{4}+2,288 \mathrm{C}_{6}-\mathrm{C}_{12}}{1,801,800}-12 h \frac{691 h^{11} v^{\mathbf{r i}}}{750,75^{j}}+\ldots(n \geqslant 12)
\end{aligned}
$$

The derivatives in the last or corrective terms of these equations may be expressed in terms of finite differences should the latter be more convenient. The transformation is well known, but its most essential features may be noted here to save a reference. The $k$ th derivative of a function, $f(x)$, multiplied by the kth power of the constant increment of $x$, here denoted by $h$, is expressible in terms of the $k$ th finite difference and differences bigher than the $k$ th. For the purpose in hand Newtonian differences should be employed, because they are applicable at the beginning and at the end of a series of values. When the derivatives and differences are so large that higher derivatives and differences also require consideration the transformation is somewhat complex, but if the $k$ th difference is technically "small" 
so that the $(k+1)$ st difference is negligible, then

$$
h_{h}^{h}\left(\stackrel{(k)}{h)}=\Delta^{(k)}\right.
$$

where $\Delta$ denotes finite difference. In the formulæ $v$ is employed to indicate the difference of the derivatives at the limits of the area to be integrated, $x_{0}$ and $x_{n}$. Using a corresponding notation for the finite differences and assuming that the $(k+1)$ st difference is inconsiderable

$$
l^{k} v^{k}=\Delta_{n}^{(k)}-\Delta_{0}^{(k)}
$$

and this substitution may be made in the corrective terms of the formulæ* *

Not all of these equations are wholly new. The first term of (1) is only Simpson's rule in a new notation and if $n$ is limited to 2 it is also identical with Cotes's rule for $n=2$. Omitting the derivatives, equations (2) and (3) also coincide with Cotes's rules for $n=4$ and $n=6$, but if in these equations $n$ is taken at any multiple of 4 and 6 numbers quite distinct from Cotes's result. All of the equations can be expressed in the same form as Cotes's, but this mode of statement seems undesirable because it masks the vital fact that a reduction of the value of $h$ increases the accuracy of the result. Now no one would think of getting a considerable quadrature by Simpson's rule with the minimum value of $n=2$, because this rule with $n=10$ gives a result the error of which approaches a 625 th of that incurred by taking $n$ at 2 , while if in (6) $n$ is taken at 24 instead of 12 the error is reduced approximately to $1 / 4096$ of its maximum value.

So fur as I know, equations (4), (5), and (6) are new, and Cotes's numbers for $n=8$ and 10 do not fit into the system of quadratures here discussed.

The derivative term in each of the six equations may exceed the value of the remainder. If the difference of derivatives in this term is denoted by $v^{r}$, this is to be regarded as the definite integral of $\stackrel{(r+1)}{f^{\prime}(x)}$ which, like any other function of real variables to be integrated, must preserve the same sign between the limits of jntegration. If the sign does not chiange and if also (as Poisson and Jacobi showed) the $(r+1)$ st derivative does not pass through a maximum between the limits, then the final term of the equations exceeds in absolute value the remainder of the series. In

* A discussion of the relations subsiating betwen derivatives and finite differences may be found in Smithsonian Math. Tables, 1908, page $x \times x$ vi, or elsewhere. 
any case whatever let $\lambda$ be the maximum value of $\stackrel{(r+1)}{f(x)}$ between the limits, then the total area represented by the definite integral, $v^{r}$, must be less than $\lambda\left(x_{n}-x_{0}\right)$, and this substituted for $v^{r}$ in the corrective term gives a quantity greater than the remainder of the Eulerian series. It is usually practicable so to select or subdivide the limits of integration that the $(r+1)$ st derivative neither changes sign nor passes through a maximum, and then the corrective term of the equations approximately defines the error of the quadrature.

It is possible still further to reduce the limits of the remainder provided that certain assumptions are made with respect to succeeding differential coefficients, but this proviso implies an inquiry which in most instances would be laburinus, more so than the division of $h$ into two or more jarts *.

It will be observed that even in (6) the coefficients are numbers of manageable magnitude not exceeding 7 places. If the altempt were made to eliminate a larger number of derivative terms it is not improbable that the formulæ could be dealt with only by 10 -place logarithms or computing machines.

The most accurate of the equations given abore involves the division of $x_{n}-x_{0}$ into some multiple of 12 parts.

Such a division may be inconvenient, for example in dealing with a function which is already tabulated to a decimal argument. This difficulty, however, may be avoided with little trouble; for, if 100 values of $y$ are available, the quadrature from the first to the 96 th may be effected by equation (6) and that of the remaining 4 items by equation

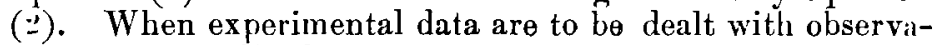
tions can usually be so arranged as to fit a duodecimal formula, and when time is the independent variable duodecimal division is of course most convenient.

In dealing with some functions one or two derivatives are readily calculated, or are perhaps already tabulated, while the higher derivatives are troublesome. It is worth while to observe, therefore, that it is as easy to eliminate the coefficients of the hirher derivatives as of the lower ones, and that a formula similar to (6) could be found in which $v^{\prime}$ and $v^{\prime \prime \prime}$ should be retained but $\boldsymbol{v}^{\mathbf{x i}}$ and $v^{\mathrm{xiii}}$ cancelled.

* The remainders in formulæ (1) to (6) arise from the remaiuders in the system of Taylor's series on which Euler's equation is based, and the publizhed discussions of the remainder in Taylor's series would make a stout volume. No elaborate consideration of this subject seerns needful for the purpose of this paper. 
Odd values of $n$ lend themselves less readily than even ones to the elimination of derivatives from Euler's series because of their limited divisibility. If $n=3$ the quadrature may be written

$$
\int_{x_{0}}^{x_{n}} y d x=\frac{9 \mathrm{C}_{1}-\mathrm{C}_{3}}{8}-3 h \frac{h^{3} v^{\prime \prime \prime}}{240},
$$

and the first term of this formula was given by Nowton*.

Comparison with (1) shows that it is somewhat less accurate than Simpson's rule. Newton's rule is derivable aiso from the integration of his interpolation formula, and if $3 h$ is taken as unity it coincides with Cotes's rule for $n=3$. The lowest odd numbor with two divisors is 9 , so that with $n=9$ two derivatives could be eliminated, but such a formula would be of small value.

It is noteworthy that the simpler rules for quadrature are derivable from any one of the three fundamental interpolation formulæ.

Other formula could be obtained by eliminating fewer coefficients than the divisibility of $n$ permits. In general that would be a waste of opportunities, but two examples are worth noting. With $n=6 \mathrm{I}$ find from $\mathrm{T}, \mathrm{C}_{2}$, and $\mathrm{C}_{3}$

$$
\int_{x_{0}}^{x_{n}} y d x=\frac{15 \mathrm{~T}+3 \mathrm{C}_{2}+2 \mathrm{C}_{3}}{20}-6 h \frac{h^{5} v^{*}}{5 \cup 40}+\ldots \ldots
$$

which is Weddle's rule with a corrective term. Under normal circumstances it is considerably less accurate than (3), as can easily be shown by applying each of the equations to the same portion of the exponential curve.

Curiously compact and accurate is a formula derived from $\mathrm{T}, \mathrm{C}_{2}, \mathrm{C}_{3}$, and $\mathrm{C}_{4}$ in which the coefficient of $\mathrm{C}_{2}$ turns out to bo zero. Of course $n$ must be 12 or a multiple thereof. It may be written

$$
\int_{x_{0}}^{x_{n}} y d x=\frac{1}{5}\left(4 \mathrm{~T}+\frac{8 \mathrm{C}_{3}-\left(\mathrm{Y}_{4}\right.}{7}\right)-12 h \frac{h^{7} v^{\mathrm{rii}}}{25,200}+. .
$$

Here the denominator of the corrective term is remarkably large or the remainder very small so that (7) may approach (6) in accuracy. Its simplicity makes it convenient for laboratory use. Economy of labour depends to some extent on the arrangement of computation, and for that reason $I$

* Opuscula, Methodus differentialis, prop. vi, Scholium. 
give in a footnote * below the details of the quadrature by (7) of a portion of the ascending exponential. It will enable the reader to perceive that no advantage is obtained by stating the formulæ in terms of the ordinates instead of the polygons. even when the division of the area to be integrated is limited to the minimum value of $n$.

It is needless to say that the integrals (1) to (6) without the corrective terms are rigorous for finite series with $n+1$ constants whose highest terms contain $x^{n}$. In any other case two distinct means exist for reducing the error of the result below a given tolerance, viz, a proper choice of the number of derivatives to be eliminated and a subsequent reduction of $h$ so far as this may be needful. Even if Euler's series ultimately becomes divergent for a given function or class of functions, the earlier part of the series is convergent and there is some term after the first at which divergence begins; in other words, the best result for a given value of $h$ is attainable by integrating a certain number

* Find $\int_{-1}^{+2 \cdot 6} e^{x} d x$ with $n=12$, or $h=0 \cdot 3$, taking values of $y$ from Smithsonian Math. Tables, by (7).

\begin{tabular}{|c|c|c|c|c|}
\hline$x$ & $y$. & T. & $\mathrm{O}_{3}$ & $\mathrm{C}_{4}$ \\
\hline $\begin{array}{lr}x_{0}=-1 \cdot 0 \\
x_{1} & -0 \cdot 7 \\
x_{2} & -0 \cdot 4 \\
x_{3} & -0 \cdot 1 \\
x_{4} & +0 \cdot 2 \\
x_{3} & 0 \cdot 5 \\
x_{8} & 0 \cdot 8 \\
x_{7} & 1 \cdot 1 \\
x_{8} & 1 \cdot 4 \\
x_{9} & 1 \cdot 7 \\
x_{10} & 2 \cdot 0 \\
x_{11} & 2 \cdot 3 \\
x_{12} & 2 \cdot 6\end{array}$ & $\begin{array}{l}y_{0} / 2 \\
y_{1} \\
y_{2} \\
y_{2} \\
y_{4} \\
y_{9} \\
y_{8} \\
y_{7} \\
y_{8} \\
y_{9} \\
y_{10} \\
y_{11} / 2 \\
y_{18} / 2\end{array}$ & $\begin{array}{l}0.496 \quad 5853 \\
0.904 \quad 8374 \\
1.648 \quad 721 \\
3.004 \quad 166 \\
5.473 \quad 947 \\
9.974 \quad 182\end{array}$ & $\begin{array}{l}0.1839397 \\
0.9048374 \\
2 \cdot 225541 \\
5.473 \quad 947 \\
6.731 \quad 869\end{array}$ & $\begin{array}{l}4 \cdot 055200 \\
67331869\end{array}$ \\
\hline $\begin{array}{l}\Sigma \\
\text { Factor ............ } \\
\text { Product }\end{array}$ & & $\begin{array}{lc}21 \cdot 502 \quad 439 \\
& 2 h \\
12 \cdot 901 & 4634 \\
= & \mathrm{T}\end{array}$ & $\begin{array}{ll}15 \cdot 5: 2 & 134 \\
& 3 h \\
13 \cdot 968 & 1206 \\
=C_{3} & \end{array}$ & $\begin{array}{ll}12 \cdot 192 & 412 \\
& 4 h \\
14 \cdot 6: 30 & 8914 \\
= & C_{4}\end{array}$ \\
\hline
\end{tabular}

These values substituted in (7) give for the answer 13.095858 . The true ralue of the last figure is 9 .

For $n=12$, but not for any multiple of $12,(7)$ can be written

$$
\begin{gathered}
\int_{x_{0}}^{x_{n}} y d x=\frac{h}{\overline{5}}\left\{\frac{10}{7}\left(y_{0}+y_{12}\right)+8\left(y_{1}+y_{3}+y_{7}+y_{11}\right)+\frac{80}{7}\left(y_{3}+y_{8}\right)\right. \\
\left.+\frac{24}{7} y_{6}-\frac{4}{7}\left(y_{4}+y_{8}\right)\right\} .
\end{gathered}
$$

[No arithmetical work is saved by adopting this method of statement.] 
of terms of Euler's series. This amounts to a chojce between formulæ (1) to (6) or others similarly derived. Thereafter the only question is how small a value of $h$ must be adopted to give the required accuracy. Any quadrature by series assumes a fictitious curve more or less nearly coinciding with a real one. For the quadratures under discussion the number of derivatives eliminated determines the order of the contact of the two curves at the extremities of the are to be integrated, and also a minimum number of common points on the two curves. By division of $i$ the number of common points is increased in simple proportion to the number by which $h$ may be divided, but the order of contact at the extremities is not affected by this process. The remainder, on the other hand, is inversely proportional to a power of the number by which $h$ is divided, a power greater by one than the order of the retained derivative.

In order to test the accuracy of formulæ for mechanical quadrature it is clearly necessary to take a difficult example, for otherwise all reasonably good formulæ would show insignificant errors. For this purpose Bertrand selected

$$
\int_{0}^{1} \frac{\log (1+x) d x}{1+x}=\frac{\pi}{8} \log 2=0 \cdot 27219826 .
$$

The curve in this case rises sharply from the origin, passes through a maximum at $x=0 \cdot 782 \overline{5} \ldots$ and then approaches the $x$-axis asymptotically. In general form it resembles the energy curve of the spectrum.

I have taken the same example using 8-place natural logarithms and an 8-figure computing machine, and get the following table of results in which I stands for the computed integral.

\begin{tabular}{|c|c|c|}
\hline Formula. & $n$. & $\mathrm{I}=\frac{\pi}{8} \log 2+$ \\
\hline 1 & 2 & +0.00181206 \\
2 & 4 & -0.00002363 \\
3 & 6 & -0.00000545 \\
4 & 8 & -0.00000154 \\
5 & 10 & -000000060 \\
6 & 12 & \pm 0.00000000 \\
7 & 12 & +0.00000001 \\
\hline
\end{tabular}

The results for (6) and (7) are very satisfactory while those for the earlier formulæ could be greatly improved by taking $n$ at a multiple of its minimum value. According to 
Bertrand, Gauss's formula for $n=4$ gives a result which is too small by 3 units in the seventh place, and thus tested it is intermediate in accuracy between (5) and (6) *

As an application of the formulæ here developed I may mention the double integral of the probability curve. By any of the more accurate formulæ (2) to (7) it may be shown that

or

$$
\begin{gathered}
\int_{0}^{\infty} d x\left[1-\frac{2}{\sqrt{ } \pi} \int_{0}^{x} e^{-x^{2}} d x\right]=\frac{1}{\sqrt{ } \pi}= \\
\int_{0}^{\infty} d x \int_{0}^{\infty} e^{-x^{2}} d x=\frac{1}{2} .
\end{gathered}
$$

In computing it 7-place values from Burgess's table were taken and the result obtained coincided with $1 / \sqrt{\pi}$ accurately to 7 places. This value is of interest in the analysis of diffusion.

Values of the probability integral itself are commonly arrived at by a somewhat intricate process, better fitted to yield a related set of values than a single one. By the formulæ given in this paper applied to tables of $e^{-x}$ isolated values of the probability integral are readily determined $\dagger$.

* Rather curionsly Weddle's sule applied to Bertrand's problera gives somewhat better results than (3). Furthermore, as this rule is denuced from Newton's interpolation formula it appears to err only by a small fraction of the sixth difference, when the seventh difference is negligible. As here deduced frum Euler's equation the error should iuclude fifth differences. While these facts are not incompatible the relations seern to need confirmation, and I have integrated $e^{x} d x$ from $x=-1$ to $x=2 \cdot 6$ taking $h=0 \cdot 6$, nd using values of $e^{x}$ with 7 significant figures. By a separate computation I find the true value of the inteyral to be 1309585 85938. Weddle's rule gives a value which is too great by 0.00064 while the value of the corrective term given in this paper for his rule is 0.00073 or about $9 / 8$ of the real error. Formula (3) rives a value 0.00016 too great or $1 / 4$ of the elror of Weddle's rule and $2 / 3$ as great as the value of the derivative term in (3).

+ For $x=0.7$, Burgess's table (Trans. R. S. Ld. vol. xxxix. 1900, p. 257, gives a value of the probability integral greater by 2 in the seventh place than that assigned to it in Jncke's table (Ast. Jahrbuch, Berlin, für 18.34). This is founded on Kramp's table (Analyse des refractions astronomique et terrestres, Leipsic et Paris, an VII. [17997) and has been adopted by Airy, Kelvin, and others. A physicist not fimiliar with the history of these tables might wish to ascertain which value is correct, and this may be accomplished by the help of (5). With $h=0.07$, seven-place values of $y=e^{-x^{2}}$ may be taken out of Smithsonian Math. Tables. Integrating by (5) and multiplying by $2 / \sqrt{ } \pi$ gives the required integral at 0.6778012 which is Burgess's value. If a computing machine is available, the arithmetical work is so more extended than in the example worked out in a previous footnote, and it requires no mathenutical knowledge beyond that required for interpolation to one additional place in the table of the exponential, so that a school-boy can do this "sum" on a single page of note-paper. 
To many experimental physicists calculus is a thorn in the flesh and $a$ weariness to the spirit, partly no doubt because they so constantly have to deal with functions whose exact mathematical expression is unknown or uncertain. I take leave to suggest that meclianical quadratures may serve them to reach conclusions which mathematicians would obtain more elegantly but in a less obrious way.

$B$ ut it appears to me that there is also room for a methodical examination by mathematicians of the applicability of formulæ such as are developed in this paper to the integration of functions which calnot be integrated in "finite terms." In such an inquiry the main point would be to determine for each class of functions the limits of convergence of Euler's series and the nature of the substitutions most conducive to increase in convergence. I hope somebody may pursue the matter further.

Washington, D.C.

April, 1911.

XXXIV. On the Ratios which the Amounts of Substances in Radioactive Equilibrium bear to one another.

To the Editors of the Philosophical Magazine.

\section{Gentlemen,- -}

ON page 40 of the last volume of this Magazine $\mathrm{Mr} . \mathrm{H}$. Mitchell has given a general solution of the system of differential equations representing the course of a series of consecutive radio ctive tran-formations. This solution is then applied to the case in which the mean length of life of the primary parent substance is greater than that of any other member in the series. Mr. Mitchell's result is more general than the one usually cited, which depends on the assumption that the parent substance not only has the smallest rate of decay, but that this rate is negligible as compared with any other in the series.

It appears worth noting that the solution given by Mr. H. Mitchell is capable of still more general interpretation. For if the $m$ th substance is the longest lived, we find, by a process precisely similar to that given in the communication referred to,

$$
\frac{x_{n}}{x_{m}+x_{m+1}+x_{n+2 \ldots} \ldots+x_{n}}=\frac{\lambda_{m}}{\lambda_{n}} \text {, when } n>m,
$$

and

$$
x_{n}=0 \text {, when } n<m \text {. }
$$

Phil Mag. S. 6. Vol. 22. No. 128. Aug. 1911. 2 A 